\title{
Antenatal physical activity: Investigating the effects on postpartum depression
}

\author{
Jennifer Guida, Swathy Sundaram, Jenn Leiferman
}

Colorado School of Public Health, University of Colorado Denver, Anschutz Medical Campus, Aurora, USA

Jennifer.guida@ucdenver.edu, Swathy.sundaram@ucdenver.edu, Jenn.leiferman@ucdenver.edu

Received 8 September 2012; revised 12 October 2012; accepted 18 October 2012

\begin{abstract}
Engaging in regular moderate-intensity physical activity has been demonstrated as a successful treatment modality for both major and minor depression and as effective as pharmacologic treatments. However, less is known about the use of antenatal physical activity as a preventive modality for depression during the perinatal period. The objective of the present study was to determine if there is an association between antenatal physical activity and PPD. A cross-sectional study using the 2007-2008 Pregnancy Risk Assessment Monitoring System (PRAMS) data from Colorado and North Carolina ( $N=6026$ ) was conducted. PRAMS self-reported data are from a large randomized sample collected by the CDC that assesses maternal demographic, socio-economic, and pre-pregnancy and perinatal behaviors. Multivariable logistic regression was used to examine the relationship between antenatal sedentary behavior and PPD. Upon adjusting for maternal age, education, race, marital status, parity, stress, smoking, drinking, and prenatal care utilization, women who did not engage in antenatal physical activity were 1.34 times more likely to screen positive for PPD than women who exercised 5 or more days per week [OR 1.34; 95\% Cl: $(1.04,1.74) ; p=0.03]$. These findings suggest that not engaging in antenatal exercise may be associated with an increase risk of PPD. Further research is warranted to better understand the effects of antenatal sedentary behavior on PPD and the potential use of physical activity as a preventive modality for PPD.
\end{abstract}

Keywords: Physical Activity; Exercise; Perinatal; Depression

\section{INTRODUCTION}

Postpartum Depression (PPD) has been estimated to affect approximately $8 \%-25 \%$ of women with onset of clinical symptoms most often occurring between three and six months postpartum and lasting up to a year [1-7]. However, the prevalence rates of PPD may be underestimated as depressive symptoms show a range in severity and often go undiagnosed and untreated [8-11]. Major Depressive Disorder (MDD) disproportionally affects women aged $20-44$, coinciding with the prime reproductive years and underscoring the importance of identifying preventive and treatment modalities for PPD among this age group [12].

PPD is associated with numerous poor maternal and child health outcomes, such as perceived negative infant behaviors and substandard parenting skills [10,13-16]. Moreover, children of depressed mothers are at greater risk for emotional, developmental and health outcomes throughout childhood and adolescence [17-21], underscoring the need to identify potential preventive modalities. To date most research focuses on identifying potential treatment modalities for PPD. Standard of care treatment approaches include pharmacologic treatments (e.g. anti-depressants), cognitive therapy as well as alternative treatments (e.g. mindfulness, meditation, physical activity) [22-24]. In particular, engaging in regular Moderate-intensity Physical Activity (MPA) has been demonstrated as a successful treatment modality for major and minor depression in the general population [25] and as effective in reducing depression symptomatology as pharmacologic treatments [9]. However, less is known about the therapeutic effects of antenatal exercise on depression during the perinatal period.

The American Congress of Obstetricians and Gynecologists (ACOG) and the Department of Health and Human Services (DHHS) recommend that women with uncomplicated pregnancies engage in regular MPA during pregnancy $[26,27]$. However, MPA prevalence rates among pregnant women are lower than those of the general population and these rates tend to decrease as pregnancy progresses $[28,29]$ putting a large population at risk for pregnancy-associated risk factors [30] including gestational diabetes mellitus [22,31,32], maternal obesity 
and excessive weight gain during late pregnancy [33], and pre-eclampsia [31,34-36]. Additionally, long-term sedentary lifestyles are associated with increased risks for chronic diseases later in life such as cardiovascular disease [37,38], diabetes [39], obesity [39], and depression $[40,41]$. Given these known detrimental effects of sedentary lifestyle, the role of antenatal MPA may play a preventative role in the development of PPD.

Most of the literature to date measures MPA during the postpartum period when depressive symptoms are already present, thus examining MPA as a treatment modality. These studies show promise and support the positive effects of postnatal MPA as a treatment modality for PPD [42-47]; however measuring MPA during the postpartum period fails to include the cumulative effect of exercise throughout pregnancy, which may play a preventative role in the development of PPD.

Less is known about the use of antenatal exercise as a preventive modality for PPD. To date there are only a few known studies that have examined the effects of antenatal physical activity on PPD. In a small randomized control trial $(\mathrm{N}=67)$, Barakat et al. (2011) examined the effects of exercise throughout pregnancy (i.e. weeks 6 - 9 through weeks 38 - 39) and determined that the exercise group (i.e. engaged in 35 - 45 min of moderate-intensity exercise 3 days/wk) reported significantly "better wellbeing" than the control group. However, mood was not the primary outcome of this study and the King's Health Questionnaire was used to assess psychosocial health, which is not an established valid and reliable screening tool for PPD symptomatolgy.

In a cross-sectional study, Nordhagen and SundgotBorgen (2002) found that MPA during the third trimester was associated with lower depression scores six weeks postpartum. However, these findings are limited due to the small sample study employed, lack of controlling for potential covariates, and the measurement error related to the operational definition of physical activity. Last, another cross-sectional study using 2004-2005 North Carolina Pregnancy Risk Assessment Monitoring System Data (PRAMS), found that women who exercised before and during pregnancy had a decreased odds of PPD (OR $=0.66,95 \%$ CI: 0.49, 0.87) after adjusting for age and marital status. However, this study fails to control for other confounding variables such as demographic, socioeconomic, and certain maternal behaviors (i.e. prenatal care utilization) [48]. Additionally, the study combined women who were told not to exercise with women who chose not to engage in physical activity during pregnancy. There should be an important distinction made between these two groups of women because women who were told not to exercise were likely "atrisk" for obstetric complications, potentially biasing the results and leaving the true role of sedentary behavior unclear.

The present study builds upon these findings by looking at physical activity during the third trimester of pregnancy to determine if antenatal physical activity is associated with PPD. Moreover this work extends the work of Ersek and Huber (2009) by expanding the PRAMS sample to two states and conducting a more comprehensive analysis which examines the effects of sedentary behavior during pregnancy while controlling for numerous demographic, socio-economic, and behavioral covariates. We hypothesize that women who did not engage in antenatal physical activity (i.e. were sedentary) during the last three months of their pregnancy will have an increased risk of PPD compared to women who reported engaging in regular exercise (i.e. exercising five or more days per week during the last three months of their pregnancy), while controlling for various demographic, socioeconomic and behavioral factors.

\section{METHODS}

\subsection{Data}

The Pregnancy Risk Assessment Monitoring System (PRAMS) is a surveillance system administered by the Centers for Disease Control and Prevention (CDC) that collects state-specific population data through self-administered questionnaires. Under the CDC, states conduct random sampling on 1300 - 3400 women two to nine months after giving birth from thirty voluntary participating states. Universal core questions among the state-specific surveys allows for comparisons among states. Through an optional standard questionnaire, states may also include additional questions on their state-specific survey, making comparisons of certain additional variables more challenging across states. Oversampling of smaller, high-risk groups is conducted to ensure adequate sample sizes. PRAMS data include comprehensive variables including maternal behaviors and demographic and socioeconomic variables representing the pre-pregnancy, antenatal, and post delivery periods (CDC PRAMS, 2011).

\subsection{Study Population}

All women who had a live birth in Colorado or North Carolina in 2007 or 2008 and who were randomly selected by the CDC PRAMS survey were included in the study [8]. The source population contained 7075 mothers, with a $68 \%$ response rate from Colorado in 2007 and 2008 and a $71 \%$ and $72 \%$ response rate from North Carolina in 2007 and 2008, respectively. Figure 1 explains the study criteria. Cases were excluded if the participant was told not to exercise ( $\mathrm{N}=739$ ), or were less than 14 years old $(\mathrm{N}=6)$ due to potential age misclassification. After excluding missing data $(\mathrm{N}=304)$, the 


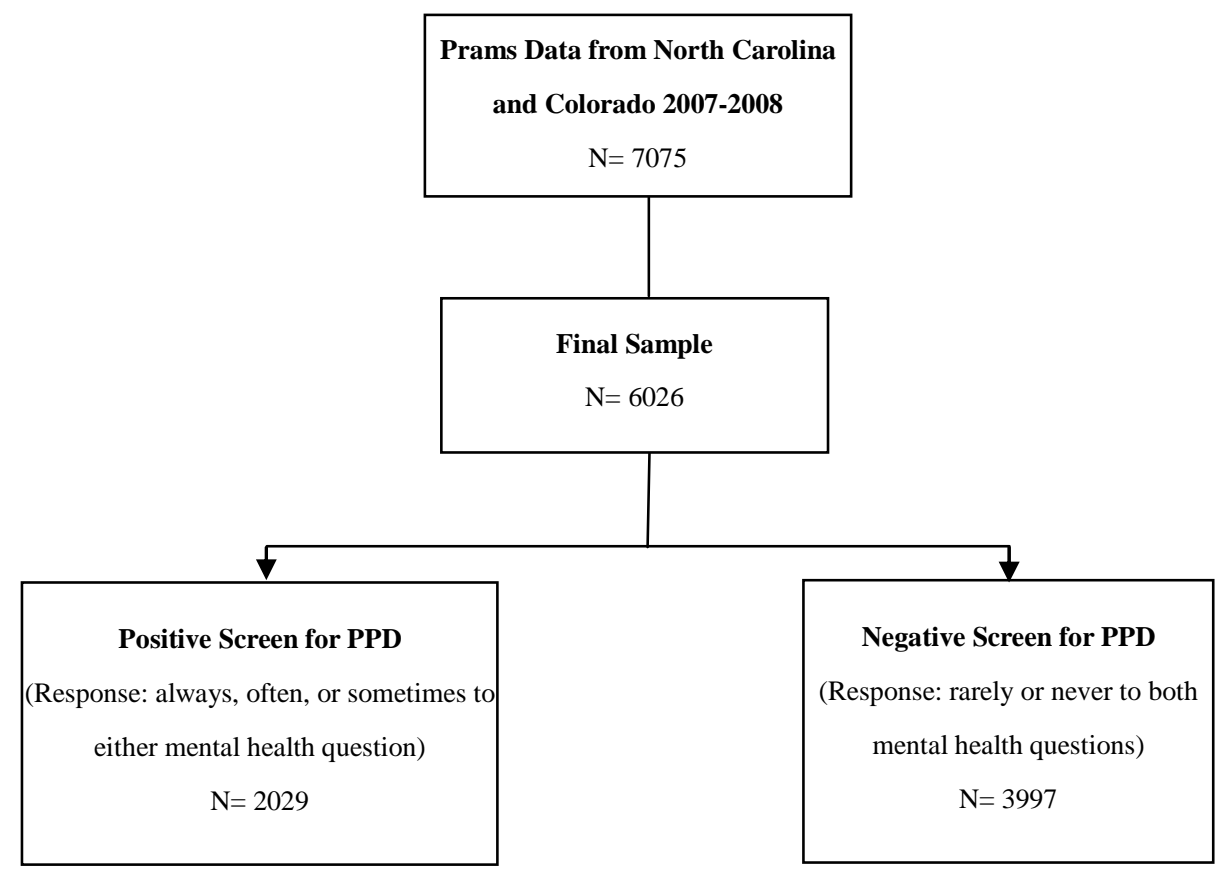

Figure 1. Exclusion criteria for the study.

final sample size contained 6026 mothers. The PRAMS survey provides weights to account for non-response bias and the sampling design, of which was taken into ac count in the analysis. This study was approved by the Colorado Multiple Institutional Review Board.

\subsection{Measures}

\subsubsection{Outcome Variable: Postpartum Depression}

PPD was measured by a two-item indicator similar to the PRIME-MD. The PRIME-MD is a well-established 2-item screening tool commonly used to assess depression risk [49]. Specifically two of the items were: "Since your new baby was born, how often have you felt down, depressed, or hopeless?" and "Since your new baby was born, how often have you had little interest or little pleasure in doing things?" Responses for each item were collapsed into dichotomous variables (i.e. always/often/ sometimes versus rarely/never), with the former considered a positive response. A positive response to either or both questions was considered a "positive screen" for PPD consistent with past research. Note that PPD throughout this paper refers to women who report high levels of depressive symptomatology and does not represent a clinical diagnosis of PPD.

\subsubsection{Primary Explanatory Variable}

Physical activity was measured by the question, "During the last 3 months of your most recent pregnancy, how often did you participate in any physical activity or exercise for 30 minutes or more?" with a response set of "less than one day per week, one to four days per week, and five or more days per week". Antenatal sedentary behavior refers to participants who engaged in less than one day of physical activity per week.

\subsubsection{Adjusted Variables}

The covariates were selected based on the previously described evidence establishing their associations with PPD $[14,25,50]$. Maternal age was grouped into the following categories: less than 20 years, women aged 20 34 , and women 35 years and older. Maternal race was coded as White, Black, Other, or Hispanic. A dichotomous marital status variable was created: married versus other (single, divorced, and widowed). Current education level of the mother was categorized into a dichotomous response of having a high school education or less and more than a high school education. The dichotomous variable parity was determined by the question "Before you got pregnant with your new baby, did you ever have any other babies who were born alive?" Responses coded "yes" were considered multipara and "no" were primipara. Maternal smoking status during pregnancy (i.e. yes/no) as well as drinking status during the last three months of pregnancy (i.e. yes/no) was also assessed.

Prenatal care Utilization (PNU) was assessed using the Kessner Index first described by the Institute of Medicine in 1973. The Kessner Index is an algorithm used to determine the adequacy of PNU based on the timing of the initiation of prenatal care and the number of prenatal care visits adjusted for gestational age. Adequate prena- 
tal care begins in the first trimester, Intermediate prenatal care starts in the second trimester, and Inadequate prenatal care starts in the third trimester or was not initiated at all [51]. The Kessner Index uses the ACOG recommendation to classify the standard for adequate number of visits.

\subsection{Data Analysis}

SAS version 9.2 was used to analyze the data. Bivariate analyses were conducted to calculate unadjusted odds ratios. Multivariable logistic regression was used to model PPD and all risk factors using a forward approach. Our best model was chosen using the AIC and the Wald Chi Square to determine overall model fit, aiming for the most parsimonious model. Covariates were considered confounders if the adjusted estimates changed by more than $10 \%$, which is widely accepted by the literature. Body Mass Index (BMI) was the only covariate that did not change our estimates by $10 \%$ and therefore was not included in our final model. All results are reported as odds ratios with $95 \%$ confidence intervals.

\section{RESULTS}

The results for the univariate analysis are presented as weighted percentages in Table 1. Approximately 33\% of women reported PPD, $44.3 \%$ of women were completely sedentary, while $45.2 \%$ reported exercising at least 1 - 4 days per week and $10.5 \%$ reported 5 or more days of exercise per week during the last three months of pregnancy. $75 \%$ of our sample was between the ages of 20 34 years old. Over half of our sample were Caucasian, had greater than a high school education, were multiparous, and reported having adequate prenatal care. See Table 1 for full sample descriptive statistics.

To further explicate these relationships, bivariate and multivariable logistic regression analyses were completed. The results for the unadjusted bivariate analysis are presented in Table 2. The unadjusted bivariate results showed that women who were sedentary had 1.36 higher odds of PPD than women who exercised 5 or more days per week during the third trimester of pregnancy (Odds Ratio $(\mathrm{OR})=1.36,95 \%$ Confidence Interval $(95 \% \mathrm{CI})$ : $1.15,1.62$, p-value $<0.0005)$. In addition, women who exercised 1 - 4 days per week had 1.10 increased odds of PPD compared to those who exercised 5 or more days per week (95\% CI: 0.93, 1.32, p-value $=0.24)$.

Multivariable analyses adjusted for the following variables: maternal age, education, race, marital status, parity, stress, prenatal care utilization, maternal smoking, and maternal drinking and are shown in Table 3. The multivariable logistic regression results supported our hypothesis that sedentary women during their last three months of pregnancy had 1.34 higher odds of PPD than women who exercised five or more days per week (95\% CI: $1.04,1.74$, p-value $=0.03$ ). In addition, women who exercised 1 - 4 days per week during their last trimester had 1.18 higher odds of PPD compared to women who exercised 5 or more days per week (95\% CI: 0.92, 1.53, p-value $=0.19)$. Women who reported PPD were more likely to have received a high school education or less (OR: 1.15, 95\% CI: 0.96, 1.38, p-value $=0.14$ ), be unmarried (OR: 1.32, 95\% CI: 1.09, 1.60, p-value = 0.005), and smoke (OR: 1.49, 95\% CI: 1.15, 1.92, p-value = 0.0025). Women with PPD had 4.34 higher odds of reporting 6 - 18 life stressors 12 months before pregnancy than women who reported none (95\% CI: 3.02, 6.24, $\mathrm{p}$-value $<0.0001)$. Additionally, women with PPD had 2.58 higher odds of reporting 3 - 5 life stressors (95\% CI: 2.08, 3.21, p-value $<0.0001$ ) and 1.48 higher odds of reporting 1 - 2 life stressors 12 months prior to pregnancy than women who reported none (95\% CI: 1.22 , 1.79 , p-value $<0.0001)$. In addition, those experiencing PPD had a 1.15 higher odds of receiving inadequate prenatal care (95\% CI: $0.85,1.55$, p-value $=0.37$ ) and a 1.21 higher odds of receiving intermediate prenatal care (95\% CI: 1.01, 1.46, p-value $=0.04)$. Women with PPD were also less likely to be primiparous (OR: 0.83, 95\% CI: 0.70, 0.98, p-value $=0.03$ ).

\section{DISCUSSION}

The primary aim of this study was to examine the effects of antenatal physical activity on PPD. As hypothesized the results suggest that women who reported sedentary behavior (i.e. less than one day of physical activeity per week) during the last three months of their pregnancy were at increased odds for PPD compared to women who engaged in regular moderate-intensity physical activity. Approximately 33\% of the women in the PRAMS sample reported PPD symptomatology. These rates more than likely reflect women who were at risk for minor and major depression and are consistent with previous research that suggests rates for major and minor depression can be as high as 50\% [48]. Regarding sedentary behavior, $44 \%$ of women in this sample were classified as being sedentary during pregnancy whereas just over half of the women (56\%) were classified as having engaged in antenatal physical activity; which is consistent with national antenatal physical activity prevalence rates ranging from 42\% - 65\% [52,53].

The present study's findings suggest that sedentary behavior during pregnancy increases the odds of PPD symptoms by approximately 34\%. These findings are in alignment with the few extant studies examining PPD or maternal wellbeing in the postpartum period. A small cross-sectional study conducted in Norway $(\mathrm{N}=203)$ found a similar relationship suggesting that women who 
Table 1. Weighted descriptive frequencies from PRAMS 2007-2008 data.

\begin{tabular}{|c|c|c|}
\hline Independent Variables & Number $N=6330$ & Weighted Percentages (\%) \\
\hline \multicolumn{3}{|l|}{ PPD } \\
\hline PPD Symptoms & 2131 & 32.9 \\
\hline No PPD Symptoms & 4199 & 67.1 \\
\hline \multicolumn{3}{|l|}{ Physical Activity } \\
\hline Less than 1 Day Per Week & 2477 & 44.3 \\
\hline 1 - 4 Days Per Week & 2950 & 45.2 \\
\hline 5 or More Days Per Week & 776 & 10.5 \\
\hline \multicolumn{3}{|l|}{ Age } \\
\hline Less than 20 Years & 642 & 10.7 \\
\hline $20-34$ Years & 4725 & 75.3 \\
\hline 35 Years or More & 963 & 14.0 \\
\hline \multicolumn{3}{|l|}{ Maternal Education } \\
\hline High School Education or Less & 2661 & 47.3 \\
\hline More than High School Education & 3606 & 52.7 \\
\hline \multicolumn{3}{|l|}{ Maternal Race } \\
\hline Caucasian & 3861 & 57.7 \\
\hline African American & 774 & 15.3 \\
\hline Hispanic & 1332 & 5.1 \\
\hline Other & 363 & 21.9 \\
\hline \multicolumn{3}{|l|}{ Marital Status } \\
\hline Married & 4408 & 65.1 \\
\hline Other & 1915 & 34.9 \\
\hline \multicolumn{3}{|l|}{ Grouped Stress } \\
\hline None & 1904 & 29.7 \\
\hline $1-2$ & 2605 & 41.7 \\
\hline $3-5$ & 1436 & 23.2 \\
\hline $6-18$ & 335 & 5.4 \\
\hline \multicolumn{3}{|l|}{ Kessner Index for Prenatal Care } \\
\hline Adequate & 4115 & 69.1 \\
\hline Intermediate & 1541 & 22.2 \\
\hline Inadequate & 513 & 6.1 \\
\hline Unknown & 161 & 2.6 \\
\hline \multicolumn{3}{|l|}{ Parity } \\
\hline Previous Live Birth & 3459 & 57.2 \\
\hline No Previous Live Birth & 2863 & 42.8 \\
\hline \multicolumn{3}{|l|}{ Maternal Smoking } \\
\hline Yes & 678 & 9.4 \\
\hline No & 5641 & 90.6 \\
\hline \multicolumn{3}{|c|}{ Maternal Drinking Last Three Months of Pregnancy } \\
\hline Yes & 576 & 8.5 \\
\hline No & 5659 & 91.5 \\
\hline
\end{tabular}

All variables were estimated using Chi-squared statistic. All variables were statistically significant at $\mathrm{p}<0.0001$ level, with the exception of maternal education $(\mathrm{p}=0.0018)$. 
Table 2. Weighted percentages of postpartum depression and risk factors from 2007-2008 PRAMS data.

\begin{tabular}{|c|c|c|}
\hline Independent Variables & No PPD (\%) N = 4199 & PPD (\%) N = 2131 \\
\hline \multicolumn{3}{|l|}{ Physical Activity } \\
\hline Less than 1 Day Per Week & 28.5 & $15.7^{*}$ \\
\hline 1 - 4 Days of Exercise Per Week & 31.0 & 14.2 \\
\hline 5 or More Days of Exercise Per Week & 7.5 & 3.1 \\
\hline \multicolumn{3}{|l|}{ Age } \\
\hline Less than 20 Years & 6.7 & 4.0 \\
\hline 20 - 34 Years & 51.0 & 24.3 \\
\hline 35 Years or More & 9.4 & 4.6 \\
\hline \multicolumn{3}{|l|}{ Maternal Education } \\
\hline High School Education or Less & 30.0 & $17.3^{* * * *}$ \\
\hline More than High School Education & 37.3 & 15.4 \\
\hline \multicolumn{3}{|l|}{ Maternal Race } \\
\hline Caucasian & 38.9 & 18.8 \\
\hline African American & 9.7 & 5.6 \\
\hline Hispanic & 15.2 & 6.7 \\
\hline Other & 3.3 & 1.8 \\
\hline \multicolumn{3}{|l|}{ Marital Status } \\
\hline Married & 46.4 & $18.7^{* * * * *}$ \\
\hline Other & 20.7 & 14.2 \\
\hline \multicolumn{3}{|l|}{ Grouped Stress } \\
\hline None & 23.1 & $6.6^{* * * *}$ \\
\hline $1-2$ & 29.0 & 12.7 \\
\hline $3-5$ & 12.7 & 10.5 \\
\hline $6-18$ & 2.1 & 3.3 \\
\hline \multicolumn{3}{|l|}{ Kessner Index for Prenatal Care } \\
\hline Adequate & 47.8 & $21.3^{* * *}$ \\
\hline Intermediate & 14.0 & 8.3 \\
\hline Less than Adequate & 3.6 & 2.5 \\
\hline Unknown & 1.7 & 0.8 \\
\hline \multicolumn{3}{|l|}{ Parity } \\
\hline Previous Live Birth & 29.4 & 13.4 \\
\hline No Previous Live Birth & 37.7 & 19.5 \\
\hline \multicolumn{3}{|l|}{ Maternal Smoking } \\
\hline Yes & 4.7 & $4.7^{* * * *}$ \\
\hline No & 62.3 & 28.3 \\
\hline \multicolumn{3}{|c|}{ Maternal Drinking Last Three Months of Pregnancy } \\
\hline Yes & 5.7 & 2.8 \\
\hline No & 61.2 & 30.3 \\
\hline
\end{tabular}

All variables were estimated using Chi-squared statistic. $\mathrm{p}$-values are denoted as ${ }^{*} \mathrm{p}<0.05 ;{ }^{* *} \mathrm{p}<0.01 ; \stackrel{* * *}{\mathrm{p}}<0.001 ;{ }^{* * * * *} \mathrm{p}<0.0001$. 
Table 3. Multivariable logistic regression results for the unadjusted and adjusted model using 2007-2008 PRAMS data.

\begin{tabular}{|c|c|c|c|}
\hline \multirow[b]{2}{*}{ Independent Variables } & \multicolumn{3}{|c|}{ Model 1} \\
\hline & Unadjusted OR & Adjusted OR & $95 \%$ CI \\
\hline \multicolumn{4}{|c|}{ Physical Activity (5 or More Days Per Week) } \\
\hline Less than 1 Day Per Week & $1.36^{* * *}$ & $1.34^{*}$ & $(1.04,1.74)$ \\
\hline 1 - 4 Days Per Week & 1.10 & 1.18 & $(0.92,1.53)$ \\
\hline \multicolumn{4}{|l|}{ Age (20 - 34) } \\
\hline Less than 20 Years & & 0.97 & $(0.73,1.30)$ \\
\hline 35 Years or Older & & 1.17 & $(0.94,1.45)$ \\
\hline \multicolumn{4}{|c|}{ Maternal Education (More than High School) } \\
\hline High School Education or Less & & 1.15 & $(0.96,1.38)$ \\
\hline \multicolumn{4}{|l|}{ Maternal Race (Caucasian) } \\
\hline African American & & 0.88 & $(0.69,1.12)$ \\
\hline Hispanic & & 0.82 & $(0.66,1.01)$ \\
\hline Other & & 1.14 & $(0.82,1.59)$ \\
\hline \multicolumn{4}{|l|}{ Marital Status (Married) } \\
\hline Other & & $1.32^{* *}$ & $(1.09,1.60)$ \\
\hline \multicolumn{4}{|l|}{ Parity (Multipara) } \\
\hline Primipara & & $0.83^{*}$ & $(0.70,0.98)$ \\
\hline \multicolumn{4}{|l|}{ Smoking (No) } \\
\hline Yes & & $1.49^{* *}$ & $(1.15,1.92)$ \\
\hline \multicolumn{4}{|c|}{ Drinking during the Last 3 Months of Pregnancy (No) } \\
\hline Yes & & 1.12 & $(0.86,1.45)$ \\
\hline \multicolumn{4}{|l|}{ Grouped Stress } \\
\hline $1-2$ & & $1.48^{* * * *}$ & $(1.22,1.79)$ \\
\hline $3-5$ & & $2.58^{* * * * *}$ & $(2.08,3.21)$ \\
\hline $6-18$ & & $4.34^{* * * * *}$ & $(3.02,6.24)$ \\
\hline \multicolumn{4}{|l|}{ Kessner Index (Adequate) } \\
\hline Inadequate & & 1.15 & $(0.85,1.55)$ \\
\hline Intermediate & & $1.21^{*}$ & $(1.01,1.46)$ \\
\hline Unknown & & 1.05 & $(0.64,1.72)$ \\
\hline
\end{tabular}

All variables were estimated using Wald Chi-squared statistic. $\mathrm{p}$-values are denoted as ${ }^{*} \mathrm{p}<0.05 ;{ }^{* *} \mathrm{p}<0.01 ;{ }^{* * *} \mathrm{p}<0.001 ;{ }^{* * * *} \mathrm{p}<0.0001$.

engaged in less than 60 minutes of moderate-intensity exercise per week during pregnancy were more likely to report depressive symptomatology during the postpartum period. Additionally, studies examining maternal wellbeing show similar trends. For example, Whatley Blum et al., (2004), conducted a small, observational study ( $\mathrm{N}$ = 92) and found that women who maintained or increased physical activity from the pre-pregnancy to the postpartum period had improved maternal well-being compared to women who were sedentary or who de- creased physical activity during this time. Moreover, results from Barakat et al. (2011) suggest that women who engage in regular moderate-intensity exercise during pregnancy have an improved perception of health status, including a heightened sense of wellbeing in the postpartum period. The present study builds upon these aforementioned findings as well as extends Ersek and Huber's 2009 study by analyzing a large random sample of 6026 women representing two states, Colorado and North Carolina, while also controlling for various 
demographic, socioeconomic and behavior factors.

Consistent with previous research, being single, multiparous, and reporting moderate to high levels of perceived stress increased the odds of reporting PPD symptomatology [38,50,54-56]. Moreover, women who reported smoking during pregnancy were more likely to report PPD symptomatology than their non-smoking counterparts $[50,55,56]$. Smoking during pregnancy has been previously established as associated with being multiparous, pregnancy unwantedness and current or previous diagnoses of depression [57]. In a recent study, smoking relapse 24 weeks postpartum was attributed to negative emotions [57], however the relationship between antenatal sedentary behavior and smoking on PPD is understudied and remains unclear.

What are some potential bio-physiological mechanisms that may help explain physical activity's effect on treating and potentially preventing PPD? Endorphins, cortisol reactivity and immune functioning are a few potential mechanisms. The release of plasma endorphins after exercise and its association with enhanced mood is well established [58] however, its influence on PPD has yet to be examined. Cortisol reactivity which is influenced by the hypothalamic-pituitary-adrenal axis is another potential pathway. Typically when an individual encounters an emotional or physiological stressor, the hypothalamus is activated and releases CorticotropinReleasing Hormone (CRH), which triggers the production of Adrenocorticotropin releasing Hormone (ACTH) and then acts on the adrenal glands to produce glucocorticoids which in turn, inhibits the production of $\mathrm{CRH}$ and ACTH via a negative feedback mechanism. This is called the Hypothalamu-Pituitary-Adrenal (HPA) axis. Cortisol is a reliable indicator of HPA axis functioning and it is well established that cortisol levels are higher among depressed individuals [12,59]. However, research examining the effects of exercise on cortisol in the general population and among depressed individuals is equivocal to date $[60,61]$. Last, immune functioning markers (i.e. cytokines) have been implicated as a contributing factor in the development of depression. In particular, proinflammatory cytokines tend to be higher in depressed individuals than nondepressed individuals [62]. Recent research suggests that exercise may affect cytokine production among depressed individuals [63] but more research is warranted to better understand these potential mechanistic pathways. It should also be noted that various psychological mechanisms may also influence these relationships such as self-esteem, self-efficacy, and behavioral activation [42].

\section{LIMITATIONS}

These data are limited by self-reported measures, although past research has found self-reported data to be highly correlated with actual health behaviors [64]. Data on depressive symptomatology only represented women who were "at-risk for depression" and were not based on clinical diagnoses. Moreover, depressive symptomatology data were only collected during the postpartum period so unfortunately we were unable to discern neither if mothers experienced high levels of depressive symptommatology during pregnancy nor were we able to identify women who were actively seeking treatment for their symptoms at any time during, before, or after pregnancy. Only two states asked questions regarding both physical activity status and PPD; therefore, our sample is regionally limited and should not be generalized outside of this population. Unfortunately, the current PRAMS data also did not afford an opportunity to examine postpartum physical activity patterns so we were unable to disentangle the effects of antenatal physical activity and postpartum physical activity on PPD. However, research suggests that individuals who are sedentary pre-pregnancy are more likely to remain sedentary in the postpartum period [29]. Finally, given the cross-sectional study design, this study can only suggest an association between variables and PPD and not causality.

\section{RECOMMENDATIONS}

PPD is an understudied area of maternal and child health that has serious repercussions to both the family and the mother/child interaction [10,13-16]. Women who suffer from PPD are more likely to have subsequent depressive episodes later in life [65] underscoring the importance of identifying preventive modalities, such as potentially antenatal physical activity. Antenatal physical activity likely affects multiple bio-physiological pathways [14] and could benefit numerous women postpregnancy and later in life. Moreover, sedentary behavior is likely detrimental to health in many aspects. Despite the fact that ACOG and DHHS both purport the benefits of antenatal physical activity and encourage all pregnant women who are free of obstetric complications to engage in regular moderate-intensity physical activity, many pregnant women remain sedentary. In fact, physical activity prevalence rates are considerably lower among pregnant women compared to nonpregnant women and physical activity levels tend to decline throughout pregnancy [29,53]. Given the numerous benefits associated with antenatal physical activity [14] coupled with the lower prevalence rates for antenatal physical activity, identifying ways to increase antenatal physical activity and reduce sedentary behavior is of upmost importance.

\section{ACKNOWLEDGEMENTS}

The research team would like to acknowledge the CDC PRAMS working group for their permission to use this dataset. This study was 
conducted for a Master's Capstone project and no funding was provided. Additionally, we would like to thank Dennis Lezotte, PhD, Marci Sontag, PhD, and Eryn Callihan, MPH for their contributions.

\section{REFERENCES}

[1] Gaynes, B.N., Gavin, N., Meltzer-Brody, S., Lohr, K.N., Swinson, T., Gartlehner, G., Brody, S. and Miller, W.C. (2005) Perinatal depression: Prevalence, screening accuracy, and screening outcomes. Evidence Report-Technology Assessment (Summary), 119, 1-8.

[2] Lanes, A., Kuk, J.L. and Tamim, H. (2011) Prevalence and characteristics of postpartum depression symptomatology among Canadian women: A cross-sectional study. BMC Public Health, 11, 302. doi:10.1186/1471-2458-11-302

[3] Kendell, R.E., Chalmers, J.C. and Platz, C. (1987) Epidemiology of puerperal psychoses. The British Journal of Psychiatry: The Journal of Mental Science, 150, 662673.

[4] Munk-Olsen, T., et al. (2006) New parents and mental disorders: A population-based register study. JAMA: The Journal of the American Medical Association, 296, 25822589. doi:10.1001/jama.296.21.2582

[5] Banti, S., et al. (2011) From the third month of pregnancy to 1 year postpartum. Prevalence, incidence, recurrence, and new onset of depression. Results from the perinatal depression-research \& screening unit study. Comprehensive Psychiatry, 52, 343-351.

doi:10.1016/j.comppsych.2010.08.003

[6] Hutto, H.F., et al. (2011) Postpartum depression among White, African American, and Hispanic low-income mothers in rural southeastern North Carolina. Journal of Community Health Nursing, 28, 41-53.

doi:10.1080/07370016.2011.539088

[7] Gjerdingen, D., et al. (2011) Changes in depressive symptoms over 0 - 9 months postpartum. Journal of Women's Health, 20, 381-386.

doi:10.1089/jwh.2010.2355

[8] Centers for Disease Control and Prevention (2009) PRAMS 2009. http://www.cdc.gov/prams/

[9] Blumenthal, J.A., et al. (2007) Exercise and pharmacotherapy in the treatment of major depressive disorder. Psychosomatic Medicine, 69, 587-596. doi:10.1097/PSY.0b013e318148c19a

[10] Daley, A., Jolly, K. and MacArthur, C. (2008) The effectiveness of exercise in the management of post-natal depression: Systematic review and meta-analysis. Family Practice, 26, 154-162.

[11] Nierop, A., et al. (2006) Are stress-induced cortisol changes during pregnancy associated with postpartum depressive symptoms? Psychosomatic Medicine, 68, 931-937. doi:10.1097/01.psy.0000244385.93141.3b

[12] Handwerger, K. (2009) Differential patterns of HPA activity and reactivity in adult posttraumatic stress disorder and major depressive disorder. Harvard Review of Psychiatry, 17, 184-205.

\section{doi:10.1080/10673220902996775}

[13] Alexander, J.L. (2007) Quest for timely detection and treatment of women with depression. Journal of Managed Care Pharmacy: JMCP, 13, S3-S11.

[14] Pivarnik, J.M., et al. (2006) Impact of physical activity during pregnancy and postpartum on chronic disease risk. American College of Sports Medicine, 38, 989-1006.

[15] Nierop, A., et al. (2006) Prolonged salivary cortisol recovery in second-trimester pregnant women and attenuated salivary alpha-amylase responses to psychosocial stress in human pregnancy. The Journal of Clinical EndoCrinology and Metabolism, 91, 1329-1335. doi:10.1210/jc.2005-1816

[16] Paulson, J.F. and Bazemore, S.D. (2010) Prenatal and postpartum depression in fathers and its association with maternal depression: A meta-analysis. JAMA: The Journal of the American Medical Association, 303, 19611969. doi:10.1001/jama.2010.605

[17] van der Pompe, G. (1999) The effect of depressive symptomatology on plasma cortisol reponses to acute bicycle exercise among post-menopausal women. Psychiatry Research, 85, 113-117.

[18] Zannad, F., et al. (1999) Incidence, clinical and etiologic features, and outcomes of advanced chronic heart failure: The epical study. Epidemiologie de l'insuffisance cardiaque avancee en lorraine. Journal of the American College of Cardiology, 33, 734-742. doi:10.1016/S0735-1097(98)00634-2

[19] Fremont, A.M., Correa-de-Araujo, R. and Hayes, S.N. (2007) Gender disparities in managed care: It's time for action. Women's Health Issues: Official Publication of the Jacobs Institute of Women's Health, 17, 116-119. doi:10.1016/j.whi.2007.04.001

[20] Pai, M., Zwerling, A. and Menzies, D. (2008) Systematic review: T-cell-based assays for the diagnosis of latent tuberculosis infection: An update. Annals of Internal Medicine, 149, 177-184.

[21] Annemans, L., et al. (2004) A health economic model to assess the long term effects and cost-effectiveness of PEG IFN alpha-2a in hepatitis $C$ virus infected patients. Acta Gastro-Enterologica Belgica, 67, 1-8.

[22] Barakat, R., et al. (2011) Exercise during pregnancy improves maternal health perception: A randomized controlled trial. American Journal of Obstetrics and Gynecology, 204, 402.e1-402.e7. doi:10.1016/j.ajog.2011.01.043

[23] Al., G.E. (2010) Physical inactivity among adults with diabetes mellitus and depressive symptoms: Results from two independent national health surveys. General Hospital Psychiatry, 32, 570-576.

[24] Jonsdottir, I.H., et al. (2010) A prospective study of leisure-time physical activity and mental health in Swedish health care workers and social insurance officers. Preventive Medicine, 51, 373-377. doi:10.1016/j.ypmed.2010.07.019

[25] Dunn, A.L., Trivedi, M.H. and O’Neal, H.A. (2001) Physical activity dose-response effects on outcomes of depression and anxiety. Medicine and Science in Sports and 
Exercise, 33, S587-S597. doi:10.1097/00005768-200106001-00027

[26] ACOG Committee Obstetric Practice (2002) Exercise during pregnancy and the postpartum period. Obstetrics \& Gynecology, 99, 171-173.

[27] Evenson, K.R. and Wen, F. (2010) National trends in selfreported physical activity and sedentary behaviors among pregnant women: NHANES 1999-2006. Preventive Medicine, 50, 123-128. doi:10.1016/j.ypmed.2009.12.015

[28] Yeo, S., et al. (2010) Exploratory analysis of exercise adherence patterns with sedentary pregnant women. Nursing Research, 59, 280-287. doi:10.1097/NNR.0b013e3181dbbd61

[29] Pereira, M.A., et al. (2007) Predictors of change in physical activity during and after pregnancy: Project Viva. American Journal of Preventive Medicine, 32, 312-319. doi:10.1016/j.amepre.2006.12.017

[30] Petersen, A.M., Leet, T.L. and Brownson, R.C. (2005) Correlates of physical activity among pregnant women in the United States. Medicine and Science in Sports and Exercise, 37, 1748-1753. doi:10.1249/01.mss.0000181302.97948.90

[31] Hegaard, H.K., et al. (2007) Leisure time physical activity during pregnancy and impact on gestational diabetes mellitus, pre-eclampsia, preterm delivery and birth weight: A review. Acta Obstetricia et Gynecologica Scandinavica, 86, 1290-1296. doi:10.1080/00016340701647341

[32] Jovanovic, L. (2001) What is so bad about a big baby? Diabetes Care, 24, 1317-1318. doi:10.2337/diacare.24.8.1317

[33] Clapp, J.F., 3rd and Little, K.D. (1995) Effect of recreational exercise on pregnancy weight gain and sub-cutaneous fat deposition. Medicine and Science in Sports and Exercise, 27, 170-177. doi:10.1249/00005768-199502000-00004

[34] Kelley, G.A. and Kelley, K.S. (1999) Aerobic exercise and resting blood pressure in women: A meta-analytic review of controlled clinical trials. Journal of Women's Health \& Gender-Based Medicine, 8, 787-803. doi:10.1089/152460999319110

[35] Mayer-Davis, E.J., et al. (1998) Intensity and amount of physical activity in relation to insulin sensitivity: The insulin resistance atherosclerosis study. JAMA: The Journal of the American Medical Association, 279, 669-674. doi:10.1001/jama.279.9.669

[36] Sorensen, T.K., et al. (2003) Recreational physical activity during pregnancy and risk of preeclampsia. Hypertension, 41, 1273-1280. doi:10.1161/01.HYP.0000072270.82815.91

[37] Kannel, W.B., et al. (1967) The relation of adiposity to blood pressure and development of hypertension. The Framingham study. Annals of Internal Medicine, 67, 4859.

[38] O’hara, M.W. and Swain, A.M. (1996) Rates and risk of postpartum depression-A meta-analysis. International Review of Psychiatry, 8, 37-54. doi:10.3109/09540269609037816

[39] Knowler, W.C., et al. (2002) Reduction in the incidence of type 2 diabetes with lifestyle intervention or metformin. The New England Journal of Medicine, 346, 393-403. doi:10.1056/NEJMoa012512

[40] Melzer, K., et al. (2010) Prepregnancy body mass index and resting metabolic rate during pregnancy. Annals of Nutrition \& Metabolism, 57, 221-227. doi:10.1159/000322369

[41] Melzer, K., et al. (2010) Physical activity and pregnancy: Cardiovascular adaptations, recommendations and pregnancy outcomes. Sports Medicine, 40, 493-507. doi:10.2165/11532290-000000000-00000

[42] Daley, A. (2008) Exercise and depression: A review of reviews. Journal of Clinical Psychology in Medical Settings, 15, 140-147. doi:10.1007/s10880-008-9105-Z

[43] Da Costa, D., et al. (2009) A randomized clinical trial of exercise to alleviate postpartum depressed mood. Journal of Psychosomatic Obstetrics and Gynaecology, 30, 191200. doi:10.1080/01674820903212136

[44] Craig, M. and Howard, L. (2009) Postnatal depression. Clinical Evidence, 2009, 1407.

[45] Norman, E., et al. (2010) An exercise and education program improves well-being of new mothers: A randomized controlled trial. Physical Therapy, 90, 348-355. doi:10.2522/ptj.20090139

[46] Armstrong, K. and Edwards, H. (2003) The effects of exercise and social support on mothers reporting depressive symptoms: A pilot randomized controlled trial. International Journal of Mental Health Nursing, 12, 130-138. doi:10.1046/j.1440-0979.2003.00229.x

[47] Demissie, Z., et al. (2011) Associations between physical activity and postpartum depressive symptoms. Journal of Women's Health, 20, 1025-1034. doi:10.1089/jwh.2010.2091

[48] Ersek, J.L. and Brunner Huber, L.R. (2009) Physical activity prior to and during pregnancy and risk of postpartum depressive symptoms. Journal of Obstetric, Gynecologic, and Neonatal Nursing: JOGNN/NAACOG, 38, 556-566.

[49] Spitzer, R.L., et al. (2000) Validity and utility of the PRIME-MD patient health questionnaire in assessment of 3000 obstetric-gynecologic patients: The PRIME-MD patient health questionnaire obstetrics-gynecology study. American Journal of Obstetrics and Gynecology, 183, 759-769. doi:10.1067/mob.2000.106580

[50] McCoy, S.J., et al. (2006) Risk factors for postpartum depression: A retrospective investigation at 4-weeks postnatal and a review of the literature. The Journal of the American Osteopathic Association, 106, 193-198.

[51] Kotelchuck, M. (1994) An evaluation of the kessner adequacy of prenatal care index and a proposed adequacy of prenatal care utilization index. American Journal of Public Health, 84, 1414-1420. doi:10.2105/AJPH.84.9.1414

[52] Zhang, J. and Savitz, D.A. (1996) Exercise during pregnancy among US women. Annals of Epidemiology, 6, 5359.

[53] Evenson, K.R., Savitz, D.A. and Huston, S.L. (2004) Leisure-time physical activity among pregnant women in the US. Paediatric and Perinatal Epidemiology, 18, 400407. doi:10.1111/j.1365-3016.2004.00595.x 
[54] Goodman, J.H. (2004) Postpartum depression beyond the early postpartum period. Journal of Obstetric, Gynecologic, and Neonatal Nursing: JOGNN/NAACOG, 33, 410420.

[55] Hayes, D.K., et al. (2010) Disparities in self-reported postpartum depression among Asian, Hawaiian, and Pacific Islander women in Hawaii: Pregnancy Risk Assessment Monitoring System (PRAMS), 2004-2007. Maternal and Child Health Journal, 14, 765-773. doi:10.1007/s10995-009-0504-z

[56] Bryan, T.L., et al. (1999) Incidence of postpartum depression in Olmsted County, Minnesota. A populationbased, retrospective study. The Journal of Reproductive Medicine, 44, 351-358.

[57] Park, E.R., et al. (2009) The association of depressive, anxiety, and stress symptoms and postpartum relapse to smoking: A longitudinal study. Nicotine \& Tobacco Research: Official Journal of the Society for Research on Nicotine and Tobacco, 11, 707-714. doi:10.1093/ntr/ntp053

[58] Steinberg, H. and Sykes, E.A. (1985) Introduction to symposium on endorphins and behavioural processes; review of literature on endorphins and exercise. Pharmacology, Biochemistry, and Behavior, 23, 857-862. doi:10.1016/0091-3057(85)90083-8

[59] Dinan, T.G. (1994) Glucocorticoids and the genesis of depressive illness. A psychobiological model. The British
Journal of Psychiatry: The Journal of Mental Science, 164, 365-371.

[60] Kiive, E., et al. (2004) Growth hormone, cortisol and prolactin responses to physical exercise: Higher prolactin response in depressed patients. Progress in Neuro-Psychopharmacology \& Biological Psychiatry, 28, 1007-1013. doi:10.1016/j.pnpbp.2004.05.035

[61] van der Ploeg, H.P., et al. (2011) The role of pre-pregnancy physical activity and sedentary behaviour in the development of gestational diabetes mellitus. Journal of Science and Medicine in Sport/Sports Medicine Australia, 14, 149-152.

[62] Dantzer, R., et al. (1999) Cytokines and depression: Fortuitous or causative association? Molecular Psychiatry, $\mathbf{4}$ 328-332. doi:10.1038/sj.mp.4000572

[63] Hallberg, L., et al. (2010) Exercise-induced release of cytokines in patients with major depressive disorder. Journal of Affective Disorders, 126, 262-267.

[64] US Department of Health \& Human Services (2008) Physical activity guidelines for Americans. http://www.health.gov/paguidelines/guidelines/default.as pX

[65] Horwitz, S.M., et al. (2009) Persistence of maternal depressive symptoms throughout the early years of childhood. Journal of Women's Health, 18, 637-645. doi:10.1089/jwh.2008.1229 